\title{
Ultraviolet Light-Responsive Photorheological Fluid for Sensors and Actuators Realized by Phosphorescence Effects and LSTM RNN
}

\author{
Min Young Cho', Suman Timilsina ${ }^{2}$, Jong Wook Roh $^{2}$, Walter Commerell, \\ Ho Geun Shin ${ }^{4}$, Yong-Nam Kwon ${ }^{5 *}$, and Ji Sik Kim ${ }^{2 *}$ \\ ${ }^{1}$ Department of Automotive Engineering, Kyungpook National University, Sangju 37224, Republic of Korea \\ ${ }^{2}$ School of Nano \& Advanced Materials Engineering, Kyungpook National University, Sangju 37224, Republic of Korea \\ ${ }^{3}$ Institute of Energy and Drive Technology, Ulm University of Applied Sciences, Ulm 89075, Germany \\ ${ }^{4}$ Dept. of Advanced Science and Technology Convergence, Kyungpook National University, Sangju 37224, Republic of Korea \\ ${ }^{5}$ Department of Materials Processing, Korea Institute of Materials Science, Changwon 51508, Republic of Korea
}

\begin{abstract}
A photo-rheological fluid (PRF) is a smart fluid which exhibits different viscosity under UV irradiation. A PRF is comprehensively presented in this work, with particular focus on its responses under UV off/on conditions. The isomeric conversion from SP to MC and vice versa under UV off and on, respectively, showed unequal rates of transformation. As a result, a complex non-linear hysteretic response was observed. To be used indifferent types of sensors and actuators which can exploit its rheological properties, it is essential the PRF have linearized hysteresis behavior. To minimize the asymmetric non-linear hysteresis characteristics under UV on and off conditions, the well-known long-lasting phosphor SAO $\left(\mathrm{SrAl}_{2} \mathrm{O}_{4}: \mathrm{Eu}^{2+}, \mathrm{Dy}^{3+}\right)$ was incorporated. The incorporation of SAO in the PRF improved the linearity of the PRF response, although the conversion rate was not identical under UV off/on conditions. The SAO particles were observed to settle over time due to phase splitting, undermining the usefulness of the SAO-PRF composite. Instead of improving the PRF response by further adjusting the PRF composite, a software approach based on Long Short-Term Memory Recurrent Neural Networks (LSTM RNN) was employed to model and compensate the asymmetric non-linear hysteresis response, ensuring the realization of sensors and actuators that exploit PRF as hardware.
\end{abstract}

(Received March 2, 2021; Accepted March 5, 2021)

Keywords: smart fluid, photorheological fluid, phosphorescence, linearization, LSTM RNN

\section{Introduction}

Any fluid whose properties, such as viscosity, can be changed via certain stimuli is known as a smart fluid [1-5]. Among a wide range of smart fluids, the most studied and utilized for real applications are electro-rheological fluids (ERFs) and magneto-rheological fluids (MRFs) which are today widely used in dampers [6,7], clutches $[8,9]$, for

\footnotetext{
- 조민영·수만티밀시나: 박사후과정, 노종욱·월터코머렐·김지식: 교수, 권용남: 책 임연구원

*Corresponding Author: Ji Sik Kim

[Tel: +82-54-530-1413, E-mail: jisikkim@knu.ac.kr]

* Co-first Author: Suman Timilsina

[Tel: +82-54-530-1413, E-mail: sumanknu@hotmail.com]

* Co-Corresponding Author: Yong-Nam Kwon

[Tel: +82-55-280-3375, E-mail: kyn@kims.re.kr]

Copyright (c) The Korean Institute of Metals and Materials
}

polishing [10], brakes [11] and valves [12]. The ERFs viscosity can be altered by an applied electric field, while the MRFs viscosity can be changed by an applied magnetic field. Unfortunately, ERFs and MRFs are not single-phase solutions; consequently, particle deposition (phase splitting) is inevitable, which is the major drawback of ERFs and MRFs. Also, the necessity of high voltage for ERF and high current for MRF produces electro-magnetic interference (EMI) and consequently EMI shielding is required, another challenge to deal with. In recent years, a new type of smart fluid known as photo rheological fluids (PRFs) have been considerably studied [13-23]. As the name suggests, light plays the active role in changing the rheological properties of PRFs. This offers some advantages over the use of magnetic field and electric fields. Since the viscosity of PRFs can be changed by light, it can be altered with precision at a given 
location. The ability to precisely apply light on a given target is especially essential in nano or microscale applications [24]. Moreover, unlike ERFs and MRFs, PRFs are nanoscale structured single-phase solutions, and as a result there is no issue of phase splitting. Likewise, PRFs do not require EMI shielding or any contact components such as electrodes and electric wires. These advantages mean PRFs have great potential for use in sensors [25], microfluidics [26], microrobotics [27], lab-on-a-chip devices [28], drag-reducing fluids in recirculating systems for district heating and cooling [29], and as patternable materials [30].

Different types of PRFs have been reported based on new, original types of photosensitive organic molecules [15-29]. Most of them, including surfactants, polymers, salts, and gelators, are complex photo responsive molecules and are not readily available. However, PRFs have been developed based on simple, commercially available elements, which do not require complex synthesis. For instance, Oh et al. developed surfactant-based PRFs from a commercially available component which demonstrated a reversible many-fold rise in zero-shear viscosity under UV and visible light irradiation [31]. The commercial availability of photo responsive materials saves considerable time and effort that would otherwise be required to synthesize photo responsive molecules. As a result, scientists in both academia and industry are increasingly oriented towards inventing new applications based on commercially available photo responsive materials.

Irreversible PRFs have been also reported, but irreversibility is considered non-ideal [32]. Lee et al. has reported reversible PRFs fabricated from commercially available materials (sodium deoxycholate (SDC), lecithin, and spiropyran (SP)) where exposure to UV light increases viscosity and exposure to visible light or heat energy reduces viscosity [33]. Cho et al. employed the same PRF as a non-contact tunable damper in a cantilever beam structure [34]. Min et al. also used it and exploited its color-changing attributes under UV irradiation for a colorimetric UV sensor device [25].

Despite its simpler structure, the commercial availability of essential ingredients to facilitate its synthesis in the laboratory, its potential application in a wide range of application, and reversibility, PRFs rate of change in viscosity under UV on and off is not symmetric [26,34]. Instead, hysteresis behavior is typically observed. Linearization of the hysteresis behavior is essential before PRF can be used in different types of sensors and actuators. Understanding the hysteresis behavior and its linearization can be achieved either by mathematical modeling or by artificial intelligence-based (AI) modeling. AI-based hysteresis modeling and linearization is increasingly considered the best option to describe complex hysteresis phenomenon, which otherwise would be a challenging task using a mathematical model [35].

In this work, LSTM RNN-based real regression (RR) and linear regression (LR) methods, which are types of AI-based methods, are employed to model the response of PRF fluids under UV off/on conditions, and to linearize it, respectively. Artificial Intelligence-based method are increasingly incorporated in almost every field to overcome the inherent problems of materials [36,37]. This is the first attempt to use AI in the field of PRFs for simulation and linearization. In section 2, the basic properties of PRFs are mentioned along with their synthesis steps. The step that was taken to minimize the hysteresis effects of the PRF response with the addition of phosphor SAO nanoparticles is described in section 3-1. Section 3-2 describes the LSTM RNN-based simulation and linearization methods.

\section{Experiment Procedure}

The PRF was prepared from the materials as originally described by Lee et al. [33]. First, $1.65 \mathrm{~g}$ of bile salt sodium deoxycholate (SDC) and $7.8 \mathrm{~g}$ of phospholipid lecithin were mixed with $100 \mathrm{~mL}$ of non polar organic solvent cyclohexane. The solution was homogeneously mixed bymagnetic stirring at $65{ }^{\circ} \mathrm{C}$ until a transparent solution resulted. Thereafter, 0.48 $\mathrm{g}$ of spiropyran (SP) was added into the transparent solution and mixed by magnetic stirring at room temperature until the SP was perfectly mixed into the solution. More detailed information on the preparation of the PR fluid sample based on SP-doped reverse micelles from commercially available products is described in the literature [33].

Before UV irradiation, lecithin interacts with SDC and forms a long worm-like structure resulting in the formation of a transient network; this results in a high viscosity fluid. Meanwhile, SP, which is hydrophobic and nonionic, remains in the fluid without any interaction. As soon as the UV is turned on, SP converts into its photoisomer known as 
(a)

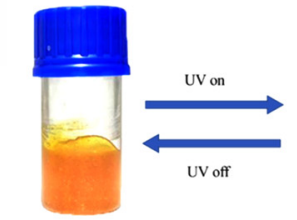

(b)

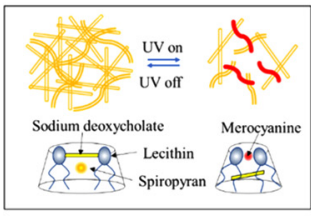

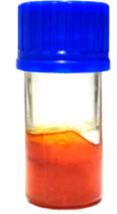

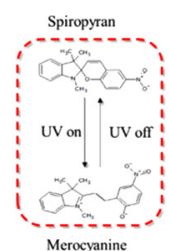

(c)

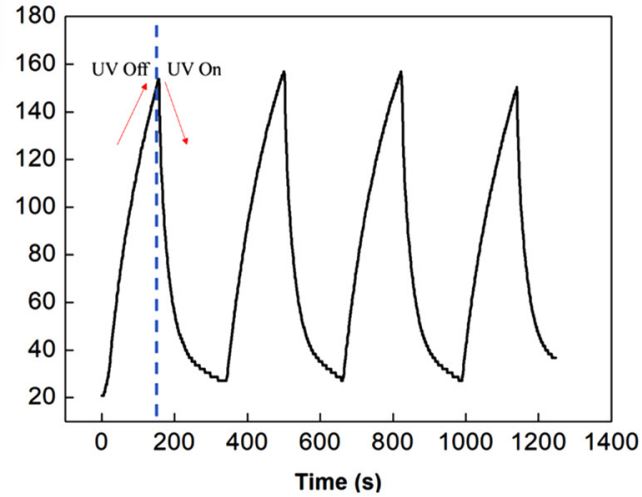

Fig. 1. Photoisomerism effect of SP on the rheological property of lecithin/SDC solution: (a) color change of PRF under UV on/condition indicating transformation of SP to MC and vice-versa (b) interaction of SP and MC with the headgroups of lecithin and illustration of chemical structure of SP and MC, (c) variation on the viscosity of the PRF composed of lecithin, SDC, and SP under UV on and off condition.

merocyanine (MC). The formed MC is zwitter-ionic and hydrophilic in nature, causing the interaction with the head groups of lecithin $[33,38,39]$. The SP and MC have different effects on the assembly of lecithin/SDC, leading to the reversible light-induced changes in the viscosity of the solution. The transformation toMC from SP is not only indicated by a decrease in viscosity but also by a change in color, from yellow to red, as illustrated in Fig. 1(a). The aforementioned changes in microstructure are illustrated in Fig. 1(b), while the measured cyclic viscosity response under light off and on is depicted in Fig. 1(b). It can be clearly seen that the viscosity before the UV is turned on has a value equal to $157 \mathrm{cP}$, while after the $\mathrm{UV}$ is on the viscosity decreased to $27 \mathrm{cP}$. This means, for a given 160 -sec exposure of UV light, the viscosity decreased 13 times. This range of viscosity was determined experimentally using UV light.

The viscosity plateau determines the maximum viscosity of the PRF.A cyclic viscosity response was determined considering the maximum and minimum viscosity value, as illustrated in Fig. 1(b). It should be noted that a constant UV source (1500 lux, INNO-CURE 200) and constant room temperature $(250 \mathrm{C})$ were maintained throughout the experiment since the SP to MC conversion depends on the intensity of the UV source as well as heat energy. The rate of isomeric conversion from SP to $\mathrm{MC}$ and $\mathrm{MC}$ to $\mathrm{SP}$ were not equivalent. In fact, the rate of $\mathrm{SP}$ to $\mathrm{MC}$ conversion exponentially decreased when the UV was on, while the MC to SP increased non-linearly when UV was removed. The entire repeatable response can be observed in Fig. 1(c).The linearly proportional conversion rate produced a symmetric and linear PRF response under UV off/on conditions, which is deemed a necessary property for the possible application of PRFs in sensors or actuators.

\section{Result and Discussion}

\subsection{Tuning the PRF rheological attributes with} the addition of phosphor SAO nanoparticles

Cho et al. added the $\mathrm{ZnS}$ :Cu phosphor to the PRF fluid and found an almost symmetrical change in viscosity occurred under UV off/on conditions. They attributed this to the effect of green emission on the SP to MC conversion and viceversa [26]. In the present work, instead of $\mathrm{ZnS}: \mathrm{Cu}$, another class of long-lasting phosphor known as $\mathrm{SAO}\left(\mathrm{SrAl}_{2} \mathrm{O}_{4}: \mathrm{Eu}^{2+}\right.$, $\mathrm{Dy}^{3+}$ ) was used to examine the performance of SAO for tailoring the rheological properties of PRF. SAO has the same peak wavelength $(520 \mathrm{~nm})$ as $\mathrm{ZnS}: \mathrm{Cu}, \mathrm{SAO}$ has certain known advantages over $\mathrm{ZnS}: \mathrm{Cu}$, such as lower density, much brighter phosphorescence, much longer phosphorescence, higher humidity resistance, and high durability [40-42]. Experiments were conducted to determine whether SAO and PRF were complementary, and whether it could be used instead of $\mathrm{ZnS}: \mathrm{Cu}$, given its certain benefits over $\mathrm{ZnS}: \mathrm{Cu}$.

A PRF containing SAO nanoparticles was prepared by mixing PRF and SAO at wt \% 100:1 using a planetary mixer. The SAO dispersed in PRF is schematically illustrated in Fig. 2(a). First, the full working range of the SAO and PRF composite was determined. The maximum and minimum 
(a)

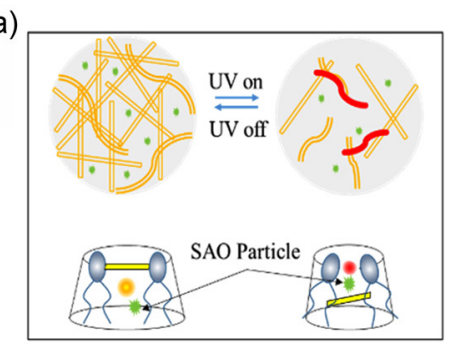

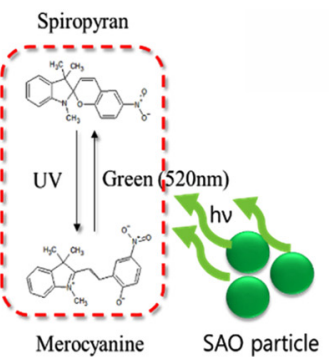

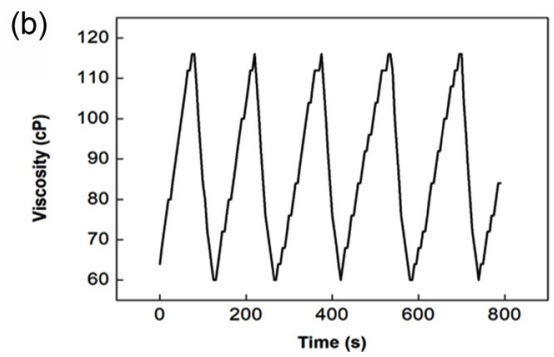

Fig. 2. (a) Dispersed SAO particle in PRF and the effect of emitted photons from SAO on SP to MC conversion and vice versa, (b) change in viscosity of the PRF composed SAO particle under UV on and off condition, which improved the linearity as well as response time.

viscosity values were determined to be $120 \mathrm{cP}$ and $60 \mathrm{cP}$, respectively. Thereafter, its cyclic viscosity response was measured under UV off and on conditions, as illustrated in Fig. 2(b). Fig. 2(b) shows that the PRF mixed with SAO phosphor exhibited an almost linear response during the UV on and off conditions. This kind of response suggests that the PRF and SAO composition is suitable for devices such as actuators and sensors. This further indicates that the intrinsic alteration of kinetic conditions in the photonic transition between SP and MC, as given in Equation (1), is linearized [26].

$$
\frac{d[S P]}{d t}=-u_{1}\left(\varphi_{S P \rightarrow M C}\right)+u_{2}\left(\varphi_{M C \rightarrow S P}\right)+u_{3}\left(\varphi_{M C \rightarrow S P}\right)
$$

where $\mathrm{u}_{1}$ stands for a chemical isomerization from SP to MC under UV. The two back-isomerizations from MC to SP is by $\mathrm{u}_{2}$ and $\mathrm{u}_{3}$ are attributed to visible chemical and thermal processes, respectively.

That is to say, the visible long green phosphorescence from the SAO particles could have accelerated the visible-chemical recovery phenomenon $(\mathrm{u} 2)$ from $\mathrm{MC}$ to SP after removing the UV irradiation. At the same time, photons from the SAO could have decelerated the SP to MC conversion during UV irradiation, thereby reducing the viscosity more or less linearly. As a result, the simple addition of SAO, made it possible to linearize the behavior of the photo-switchable fluid, like the use of $\mathrm{ZnS}: \mathrm{Cu}$.

However, the rate of change of viscosity in Fig. 2(b) suggests that the conversion rate of SP to MC was double the rate of conversion from $\mathrm{MC}$ to $\mathrm{SP}$. In order to equalize the conversion rate, further studies will be conducted in the near future considering the optimization of UV density and phosphor quantity. It should also be noted that the maximum and minimum viscosity range shrank after the incorporation of SAO. This can be attributed to the green emission during both UV on and off, which affected the conversion of SP and MC. Interestingly, for a given viscosity range, the response time was shortened by addition of SAO, as illustrated in Fig. 2 , which is a very important feature for sensors and actuator.

In the repetitive cyclic test, the SAO particles were found to settle down. To overcome this, further work considering phosphors with lower density and a porous morphology needs to be performed, so that a colloid can be formed $[43,44]$. Forming a colloidal dispersion of the phosphor particles could ensure consistent performance in sensors or actuators that exploit the photo-switchable rheological properties of the compositionally adjusted PRFs.

\subsection{LSTM RNN based simulation and linearization of the rheological response of PRF}

Even though a complex mathematical model can be developed to describe the full response of a PRF under On/ Off, AI-based methods are better than mathematical modeling since they can describe very complicated responses that might be impossible to develop mathematically $[45,46]$. The direct model of PRF response was performed using Real Regression (RR). RR is a program that learns training data obtained from real signal data and then predicts the signal from the test data. The program consists of four different parts: preprocessing the dataset, neural network modeling, learning, and testing and output. These steps were performed using the libraries of numpy, keras, matplotlib, and pandas.

For neural network modeling, LSTM RNN was employed, and its structure is illustrated in Fig. 3(a). 

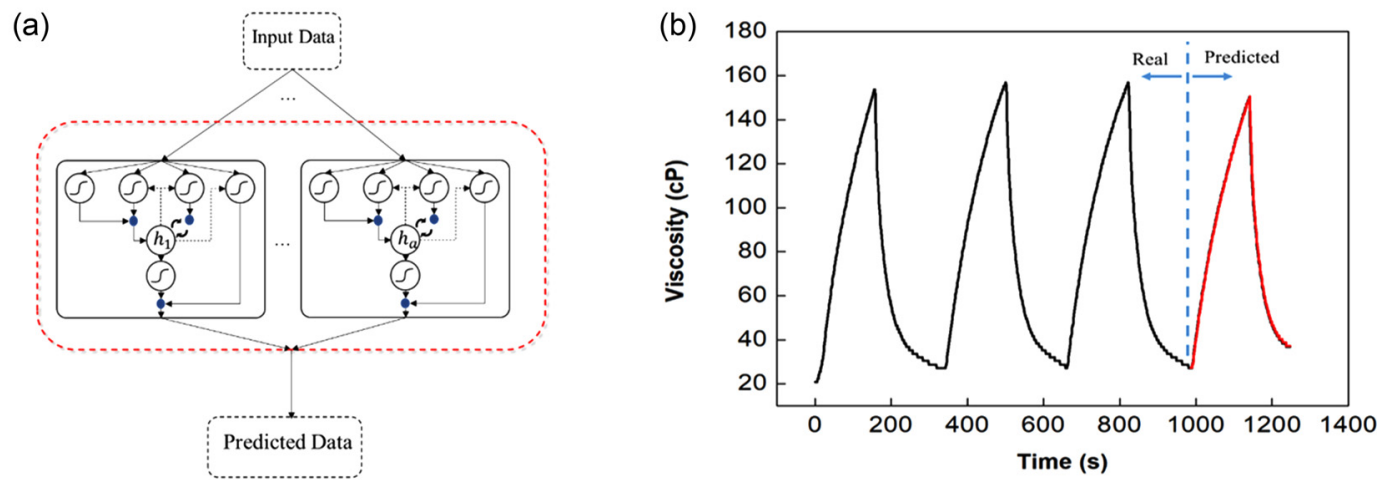

Fig. 3. (a) Structure of LSTM RNN, (b) Illustration of real data obtained from the experiment and predicted data from RR consisting of LSTM RNN.

It has 32 nodes with an input shape of (None, 1) and ReLU as an activation function. The final output layer has one node and is built with a dense layer having a linear activation function. Loss function uses L2 function (MSE) as a learning parameter of the model. Out of four PRF cyclic responses under UV on/off, three curves were used for training and the training was conducted 300 times by gradient descent. The predicted PRF cyclic response, which is indistinguishable from the real PRF cyclic response, is illustrated in Fig. 3(b). In fact, the training accuracy and test accuracy were $98.7 \%$ and $98.3 \%$ respectively. In this way, modeling of PRF cyclic response was easily performed considering the AI-based modeling route without the need to develop a mathematical model considering different parameters.

One of the most desirable properties in sensing device behavior is linearity with no hysteresis. However, sensors involved in the measurement of physical variables are often nonlinear with hysteresis behavior, and as a result they require some mechanism for hysteresis compensation. Depending on the software approach, there are different methods for this, including look-up tables, polygonal interpolation, polynomial approximation, cubic spline interpolation, and artificial neural networks (ANNs) [47]. Each of these methods has unique features, which are reflected in the software implementation, required processing resources, and the accuracy that can be achieved by it.

For the past two decades, artificial neural network (ANN)based techniques have been employed in several applications, and they have shown some success in linearization and hysteresis compensation [47-56]. In this work, linear regression (LR) was used for the hysteresis compensation of the PRF response under the UV on/off condition. LR is a program that predicts linear values after training experimentally obtained data, together with the labeling of the data. Like RR,LR also consists of four different steps and was built using the same libraries as was used for RR. The neural network was built using LSTM RNN as shown in Fig. 4(a) with 32 nodes with an input shape of $(2,1)$ and ReLU as an activation function. The final output layer has one node and was built with a dense layer having linear activation function.

The loss function known as L2 function (MSE) was used as a learning parameter in the model. First, using the data as shown in Fig. 3(b), linear data was produced by iteratively constructing the lowest and highest points of the data linearly and then linearly from the highest point to the lowest point. After loading the non-linear PRF response and linear data, the labeling data for the PRF response was produced. The total number of data shown in Fig. 3(b) was split into 4 pieces: $0 \sim 346,347 \sim 665,666 \sim 992$, and $993 \sim 1249$, where 1249 was the total number of times.

The labeling operation code means the initial labeling of a signal is zero, and the end-labeling consists of the ending number. For training, the first three cycles with their labeling were used as input data and three linear cycles were constructed as an output. Thereafter, for testing, all four of the cycles shown in Fig. 3(b) along with their labeling were used as input, which resulted in a linearized output as shown in Fig. 4(b). The compensated PRF response using LSTM $\mathrm{RNN}$ and the original PRF response are compared in Fig. 4(c), which shows good linearization except towards the 

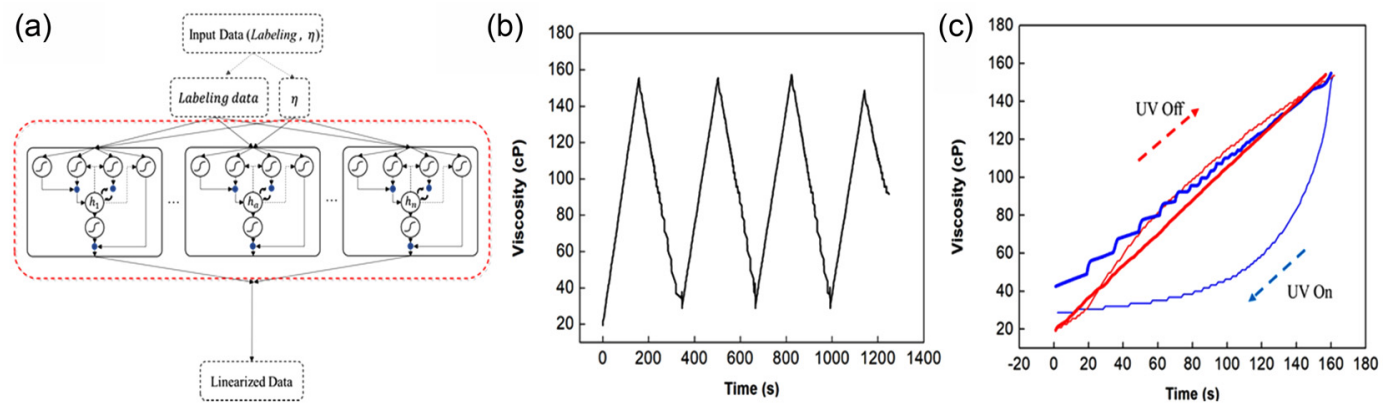

Fig. 4. (a) Structure of LSTM RNN, (b) Illustration of linearized PRF response using LR consisting of LSTM RNN, (c) Comparison of hysteresis nature of PRF and compensated PRF response.

bottom end. The differences seen towards the bottom part is because the two lowest viscosities of the response curve have slightly different values, as depicted in the hysteresis curve of the original PRF response. The complex asymmetrical nonlinear response of PRF was effortlessly simulated and linearized by employing the LSTM RNN-based neural network, without the need to consider any of the material parameters, such as relaxation time constant.

Even though the incorporating the SAO in the PRF improved its linearity, a symmetrical response was not obtained. Thus, it was interesting to employ the LSTM RNNbased neural network to address the inherent drawback of the PRF characteristics. The outcomes are very encouraging for the future employment of PRF as hardware in sensors and actuators.

\section{Conclusion}

The isomeric conversion from $\mathrm{SP}$ to $\mathrm{MC}$ and vice versa under UV off and on, respectively, exhibited unequal rates of transformation, and as a result, a complex non-linear hysteretic response was observed. To mitigate this problem, a well-known phosphor called SAO was dispersed into the PRF, which resulted in a linear response with a shorter response time. However, the PRF response was asymmetric under the UV off and on conditions. To mitigate the unsymmetrical non-linear hysteretic response, a software approach based on AI was employed. The PRF response was modeled using RR consisting of LSTM RNN, and the PRF response was symmetrically linearized using an LR consisting of LSTM RNN. The simulated PRF response was indistinguishably the same; moreover, the linearized PRF response showed good hysteretic compensation. Therefore, with the aid of AI sensors and actuators based on PRF can be realized despite the inherent hysteresis behavior of the fluid.

\section{Acknowledgment}

This work was supported by a National Research Foundation of Korea (NRF) grant funded by the Korean government (MPIS, MSIT) (No. 2015M3D1A1069719, 2021R1A2C1005732) and Fundamental \& Public Facility Building Research to Activate Industry Funding by Ministry of Science and ICT (No. POC3330) and "Human Resources Program in Energy Technology" of the Korea Institute of Energy Technology Evaluation and Planning (KETEP), granted financial resource from the Ministry of Trade, Industry \& Energy, Republic of Korea (No. 20194010000040).

\section{REFERENCE}

1. C. M. Yoon, Y. Jang, J. Noh, J. Kim, and J. Jang, ACS nano 11, 9789 (2017).

2. L. Ren, S. Sun, G. Casillas-Garcis, M. Nancarrow, G. Peleckis, M. Turdy, K. Du, X. Xu, W. Li, and L. Jiang, $A d v$. Mater. 30, 1802595 (2018).

3. M. Cao, X. Jin, Y. Peng, C. Yu, K. Li, K. Liu, and L. Jiang, Adv. Mater. 29, 1606869 (2017).

4. C. Christianson, N. N. Goldberg, D. D. Deheyn, S. Cai, and M. T. Tolley, Sci. Robot. 3, eaat1893 (2018).

5. N. Kellaris, V. G. Venkata, G. M. Smith, S. K. Mitchell, and C. Keplinger, Sci. Robot. 3, eaar3276 (2018).

6. W. W. Chooi and S. O. Oyadiji, Comput. Struct. 86, 473 
(2008).

7. N. C. Rosenfeld and N. M. Wereley, Smart Mater. Struct. 13, 1303 (2004).

8. Z. Kesy, A. Kesy, M. R. Jackson, and R. M. Parkin, Int. Rev. Mech. Eng. 2, 592 (2008).

9. A. Olszak, K. Osowski, P. Motyl, G. Mędrek, J. Zwolak, A. Kęsy, Z. Kęsy, and S. B. Choi, Front. Mater. 6, 139 (2019).

10. S. Genç and P. P.Phulé, Smart. Mater. Struct. 11, 140 (2002).

11. R. Patel, J. Magn. Magn. Mater. 2011, 1360 (2011).

12. H. Sahin, F. Gordaninejad, X. Wang, and Y. Liu, J. Intell. Mater. Syst. Struct. 23, 949 (2012).

13. Y. Z. Dong, Y. Seo, and H. J. Choi, Soft Matter 15, 3473 (2019).

14. M. Ashtiani, S. Hashemabadi, and A. Ghaffari, J. Magn. Magn. Mater. 374, 716 (2015).

15. Y. Tu, Q. Chen, Y. Shang, H. Teng, and H. Liu, Langmuir 35, 4634 (2019).

16. Y. Tu, Z. Ye, C. Lian, Y. Shang, H. Teng, and H. Liu, Langmuir 34, 12990 (2018).

17. H. Oh, A. X. Lu, V. Javvaji, D. L. DeVoe, and S. R. Raghavan, ACS Appl. Mater. Interfaces 8, 17529 (2016).

18. S. S. Babu, V. K. Praveen, and A. Ajayaghosh, Chem. Rev. 114, 1973 (2014).

19. S. Peng, Q. Guo, T. C. Hughes, and P. G. Hartley, Langmuir 30, 866 (2014).

20. A. Abdollahi, H. Roghani-Mamaqani, and B. Razavi, Prog. Polym. Sci. 98, 101149 (2019).

21. C. Zhang, Z. Liu, H. Liu, S. Wang, Y. Zhang, C. Li, and Y. Wu, Adv. Mater. Technol. 4, 201900039 (2019).

22. M. Akamatsu, M. Shiina, R. G. Shrestha, K. Sakai, M. Abe, and H. Sakai, RSC Adv. 8, 23742 (2018).

23. Y. S. Nam, I. Yoo, O. Yarimaga, I. S. Park, D. H. Park, S. Song, J. M. Kim, and C. W. Lee, Chem. Comm 50, 4251 (2014).

24. V. Gatterdam, R. Ramadass, T. Stoess, M. A. Fichte, J. Wachtveitl, A. Heckel, and R. Tampé, Angew. Chem. Int. Ed. 53, 5680 (2014).

25. K. P. Min and G. W. Kim, Sensors 19, 1128 (2019).

26. M. Y. Cho, J. S. Kim, H. J. Choi, S. B. Choi, and G. W. Kim, Smart Mater. Struct. 26, 054007 (2017).

27. S. W. Bates, Ph. D Thesis, Massachusetts Institute of Technology, Cambridge (2010).

28. S. R. Sershen, G. A. Mensing, M. Ng, N. J. Halas, D. J. Beebe, and J. L. West, Adv. Mater. 17, 1366 (2005).

29. S. Matsumoto, S. Yamaguchi, S. Ueno, H. Komatsu, M. Ikeda, K. Ishizuka, Y. Iko, K. V. Tabata, H. Aoki, and S. Ito,
Chem. Eur. J. 14, 3977 (2008).

30. H. Shi, W. Ge, H. Oh, S. M. Pattison, J. T. Huggins, Y. Talmon, D. J. Hart, S. R. Raghavan, and J. L. Zakin, Langmuir 29, 102 (2013).

31. H. Oh, A. M. Ketner, R. Heymann, E. Kesselman, D. Danino, D. E. Falvey, and S. R. Raghavan, Soft Matter 9 , 5025 (2013).

32. A. M. Ketner, R. Kumar, T. S. Davies, P. W. Elder, and S. R. Raghavan, J. Am. Chem. Soc. 129, 1553 (2017).

33. H. Y. Lee, K. K. Diehn, K. Sun, T. Che, and S. R. Raghavan, J. Am. Chem. Soc. 133, 8461 (2011).

34. M. Y. Cho, J. S. Kim, S. B. Choi, and G. W. Kim, Smart Mater. Struct. 25, 025022 (2016).

35. M. Y. Cho, J. H. Lee, S. H. Kim, J. S. Kim, and S. Timilsina, ACS Appl. Mater. Interfaces 11, 11910 (2019).

36. S. J. Jeong, I. K. Hwang, I. S. Cho, and H. S. Kim, Korean J. Met. Mater. 57, 184 (2019).

37. B.-K. Kim, N. H. Goo, J. H. Lee, and J. H. Han, Korean J. Met. Mater. 58, 822 (2020).

38. C. J. Wohl, M. A. Helms, J. O. Chung, and D. Kuciauskas, J. Phys. Chem. B 110, 22796 (2006).

39. M. Tanaka and Y. Yonezawa, J. Phys. Chem. 100, 5160 (1996).

40. J. C. Zhang, X. Wang, G. Marriott, and C. N. Xu, Prog. Mater. Sci. 103, 678 (2019).

41. A. Feng and P. F. Smet, Materials 11, 484 (2018).

42. S. Timilsina, J. S. Kim, J. Kim, and G. W. Kim, Int. J. Precis. Eng. Manuf. 17, 1237 (2016).

43. A. Huignard, T. Gacoin, and J. P. Boilot, Chem. Mater. 12, 1090 (2000).

44. M. S. Alqahtani, N. Hadia, and S. Mohamed, Appl. Phys. A 124, 617 (2018).

45. N. M. Tomasevic, A. M. Neskovic, and N. J. Neskovic, Int. J. Neural Syst. 22, 1250008 (2012).

46. N. M. Tomasevic, A. M. Neskovic, and N. J. Neskovic, Int. J. Neural Syst. 27, 1750008 (2017).

47. T. Nenov and S. Ivanov, Sens. Mater. 19, 95 (2017).

48. B. D. Lee, W. B. Park, J. W. Lee, M. S. Kim, M. H. Pyo, and K. S. Sohn. Chem. Mater. 33, 782 (2021).

49. J. W. Lee, N. H. Goo, W. B. Park, M. H. Pyo, and K. S. Sohn, Eng. Rep. 3, e12274 (2021).

50. T. Islam, Z. Uddin, and A. Gangopadhyay, Sens. Transducers 191, 126 (2015).

51. J. W. Lee, W. B. Park, B. D. Lee, S. H. Kim, N. H. Goo, and K. S. Sohn, Sci. Rep. 10, 1 (2020).

52. J. W. Lee, W. B. Park, J. H. Lee, S. P. Singh, and K. S. Sohn, 
Nat. Commun. 11, 1 (2020).

53. N. J. Medrano-Marques, and B. Martin-del-Brio, IEEE Trans. Ind. Electron. 48, 1288 (2001).

54. W. B. Park, J. Y. Chung, J. Y. Jung, K. M. Sohn, S. P. Singh, M. H. Pyo, N. S. Shin, and K. S. Sohn, IUCrJ, 4, 486
(2017).

55. J. H. Lee, S. P. Singh, M. S. Kim, M. H. Pyo, W. B. Park, and K. S. Sohn, Inorg. Chem. Front. 6, 3493 (2019).

56. C. H. Park, D. Cha, M. Kim, N. S. Reddy, and J.-T. Yeom, Met. Mater. Int. 25, 768 (2019). 